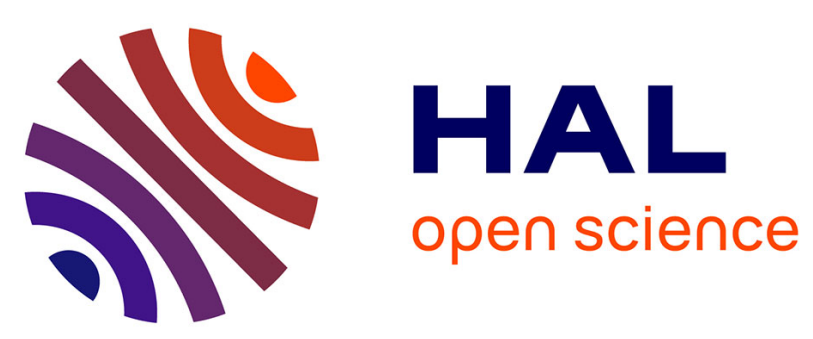

\title{
Acoustic emission from the initiation of plastic deformation of Polyethylenes during tensile tests
}

Nicolas Casiez, Stéphanie Deschanel, Thomas Monnier, O. Lame

\section{To cite this version:}

Nicolas Casiez, Stéphanie Deschanel, Thomas Monnier, O. Lame. Acoustic emission from the initiation of plastic deformation of Polyethylenes during tensile tests. Polymer, 2014, 55, pp.6561 - 6568 . 10.1016/j.polymer.2014.09.044 . hal-01113079

\author{
HAL Id: hal-01113079 \\ https://hal.science/hal-01113079
}

Submitted on 4 Feb 2015

HAL is a multi-disciplinary open access archive for the deposit and dissemination of scientific research documents, whether they are published or not. The documents may come from teaching and research institutions in France or abroad, or from public or private research centers.
L'archive ouverte pluridisciplinaire HAL, est destinée au dépôt et à la diffusion de documents scientifiques de niveau recherche, publiés ou non, émanant des établissements d'enseignement et de recherche français ou étrangers, des laboratoires publics ou privés. 


\title{
Acoustic emission from the initiation of plastic deformation of Polyethylenes during tensile tests
}

\author{
N. Casiez ${ }^{\dagger}$, S. Deschanel ${ }^{*},+$ T. Monnier ${ }^{\dagger}$ and O. Lame ${ }^{\dagger}$ \\ †Université de Lyon, MATEIS, UMR 5510, INSA de Lyon, 69621, Villeurbanne, France \\ *Université de Lyon, LVA, EA 677, INSA de Lyon, 69621, Villeurbanne, France \\ *Corresponding author: Tel (+33) 47-243-8184; Fax (+33) 47-243-8539; \\ e-mail: stephanie.deschanel@insa-lyon.fr
}

\begin{abstract}
The acoustic emission (AE) technique has been used in the aim to detect and to observe the initiation of plasticity and damage of several Polyethylenes (PE) during tensile tests. The detection of acoustic signals originating from the deformation of the material is challenging since PE samples strongly attenuate ultrasonic waves. A weak acoustic activity has been recorded during the tests. The use of two types of PE specimens and two methodologies based on the elimination and the discrimination of spurious sources has shown that most of the AE signals truly originate from the plastic deformation of materials. We also observed that the acoustic activity increases with strain rate. Besides, some AE signals are located along the specimen length during tensile tests at high strain rate. The acoustic activity increases strongly with the crystallinity. Microcavities, formed before the yield point of PE samples with high crystallinity in particular, likely initiate the release of acoustic energy. In addition, some signals are collected on PE samples, which do not exhibit a formation of cavitation. Hence, the shearing of crystallites and/or the fragmentation of crystalline lamellae may also be a source of the release of acoustic energies.
\end{abstract}

Keywords: Acoustic emission, Ultrasonic attenuation, Polyethylene, Plastic deformation, Cavitation

\section{Introduction}

The plastic deformation and damage of semi-crystalline polymers in tensile tests have been widely studied [1-13]. However, the in situ determination of the initiation of micro-structural plastic events such as shearing of crystallites or cavitation remains problematic and requires complex devices such as SAXS or tomography [9-15]. As an in situ and convenient technique, acoustic emission (AE) is often used to analyze plastic deformation and damage of materials such as metals, ceramics and composites [16-18]. AE is defined as "the class of phenomena whereby transient elastic waves are generated by the rapid release of energy from localized sources within a material, or the transient elastic waves so generated" (ASTM 1982). The plastic deformation and damage of glassy polymers have been studied with the AE technique [19-22]. Recently, Ronkay et al. [22] recorded the acoustic activity emitted from Polyethylene terephthalate (PET) during tensile tests. The authors attribute the signals to the formation of cavities, which appear with the neck propagation. However, few studies investigated the plastic deformation of pure semi-crystalline polymers above their glass transition temperature with this technique, as they are often thought to be too attenuative. Nevertheless, Bohse [23] observed a very weak acoustic activity during the plastic deformation of high-density Polyethylene (HDPE) and Polypropylene (PP) during tensile tests. Qian et al. [24] recorded few AE signals during the deformation of a large range of polymers including semi-crystalline polymers. Unfortunately, these experimental tests and analysis are insufficient to conclude about the origin of AE signals. For instance, it has not yet been possible to localize AE sources because only one sensor was used for the acquisition of the signals. Therefore, one could wonder if the AE signals recorded under these circumstances are only artifacts. However, it is interesting to note that this technique was successfully used by Galeski et al. [25,26] to collect AE signals during the spherulitic crystallization of semi-crystalline polymers from the melt. The authors attribute the 
release of acoustic waves to the formation of cavitation from the melt.

As a consequence, AE testing could be enough sensitive to analyze the initiation of plastic deformation, including cavitation, for semi-crystalline polymers above their glass transition temperature.

In this paper, we will first propose a new method to ascertain that $\mathrm{AE}$ signals recorded during tensile tests of Polyethylene samples do come from the plastic deformation and/or damage of the material. The methodologies we chose to identify the origin of sources and to analyze the AE signals will be presented. We studied several PE with different crystallinities at several strain rates in order to discuss the correlation between $\mathrm{AE}$ signals and microstructural alterations within the material.

\section{Experiments}

\subsection{Materials and samples preparation}

The five Polyethylenes (PE) examined, respectively named PE A, PE B, PE C, PE D and PE D', were obtained using the Philips method with a chromium oxide to reach a molecular weight $\mathrm{M}_{\mathrm{w}}$ equals to 231, 187, 216, 229 and $129 \mathrm{kDa}$, respectively. Their hexene C6 concentrations are equal to $1.8,0.8,0.1,0.2$ and $0 \% \cdot \mathrm{mol}$, respectively. These materials have been widely studied by Humbert et al. [9-11] and differ also by their molecular topology, which are called "linear" for the PE D', "quasi-linear" for the PE D and PE C and "branched" for the PE B and PE A. It is important to note that the PE D and PE D' samples have strongly similar characteristics, with respect to the degree of crystallinity. $6 \mathrm{~mm}$-thick plates are obtained by pellets molding between aluminium plates in a press at $170^{\circ} \mathrm{C}$ during $30 \mathrm{~min}$. Then, each PE plate has been quenched in water. This first treatment has been performed to obtain materials with a low crystallinity $\left(\chi_{c}\right)$. For this treatment, the materials will be called $P E X_{\text {que }}$ with $X=\left\{A, B, C, D\right.$ or $\left.D^{\prime}\right\}$.

A second heat treatment, named isotherm treatment, has been carried out on the PE A, PE D and PE D' samples in order to obtain different microstructural characteristics, the crystallinity in particular. This heat treatment has been performed by melting the materials at $170^{\circ} \mathrm{C}$ during $30 \mathrm{~min}$ and holding their temperature at $114^{\circ} \mathrm{C}, 123^{\circ} \mathrm{C}$ and $127^{\circ} \mathrm{C}$, respectively, in a press during 10 hours before cooling in air. For this second treatment, the materials will be named $P E X_{\text {iso }}$ with $X=\{A, D$ or $\left.\mathrm{D}^{\prime}\right\}$.

The crystallinity of PE samples has been determined by thermal analysis with a Perkin Elmer DSC7 device and calculated by the following equation at $\pm 2 \%$ :

$$
\chi_{c}=\frac{\Delta H_{f}}{\Delta H_{f}^{0}} \quad \text { (Eq. 1) }
$$

With $\Delta \mathrm{H}_{\mathrm{f}}$ the specific heat fusion of the sample and $\Delta \mathrm{H}_{\mathrm{f}}^{0}$ the heat fusion of a perfect crystal which is equal to $290 \mathrm{~J} / \mathrm{g}$ [27].

Hence, the PE samples differ from their degree of crystallinity (see Table 1), but also by their mechanisms of formation of micro-cavities:

- The PE $\mathrm{A}_{\text {que }}\left(\chi_{\mathrm{c}}=48 \%\right)$ and $\mathrm{PE} \mathrm{B}_{\text {que }}(54 \%)$ do not exhibit formation of micro voids during tensile tests;

- $\quad$ The $P E \mathrm{~A}_{\text {iso }}(51 \%), \mathrm{PE} \mathrm{C}_{\text {que }}(63 \%), \mathrm{PE} \mathrm{D}_{\text {que }}$ and PE $\mathrm{D}_{\text {que }}^{\prime}(68 \%)$ exhibit formation of microcavities which are located in the necking of samples;

- The $\mathrm{PED}_{\text {iso }}$ and $\mathrm{PED}_{\text {iso }}^{\prime}(79 \%)$ exhibit a massive formation of cavitation, which is homogenous in the center region and appears before the yield point.

It is worth noting that in this range of crystallinity plastic behavior (such as yield stress or necking) can be strongly different. As a consequence, the acoustic activity could be correlated to the 
particular plastic behavior of our materials. Indeed, it has been shown in the literature (10-13) that low crystallinity, accompanied with high "Stress Transmitters" content, delays the appearance of cavitation whereas high crystallinity enhances it. This is one of the material features that is expected to modify the acoustic activity [25].

\begin{tabular}{|c|c|c|c|c|c|}
\hline Material & Appellation & Type & Scheme & $\chi_{c}(\%)$ & Symbol \\
\hline PE A quenched & $P E A_{\text {que }}$ & \multirow{2}{*}{ «branched » } & & 48 & \\
\hline $\mathrm{PE} \mathrm{A}$ isothermed & $P E A_{i s o}$ & & & 51 & \\
\hline PE B quenched & $\mathrm{PE} \mathrm{B}_{\text {que }}$ & «branched » & & 54 & \\
\hline PE C quenched & $P E C_{\text {que }}$ & « quasi-linear » & & 63 & \\
\hline PE D quenched & $P E D_{\text {que }}$ & \multirow{2}{*}{ « quasi-linear» } & & 68 & \\
\hline PE D isothermed & $P E D_{\text {iso }}$ & & & 79 & \\
\hline PE D' quenched & PE $D_{q u e}^{\prime}$ & \multirow{2}{*}{ «linear » } & & 68 & \\
\hline PE D' isothermed & $P E D_{\text {iso }}^{\prime}$ & & & 79 & \\
\hline
\end{tabular}

Table 1. Characteristics of studied PE.

\subsection{Mechanical testing and instrumentation}

Tensile tests are carried out at three strain rates $4.2 \cdot 10^{-3} \mathrm{~s}^{-1}, 8.3 \cdot 10^{-3} \mathrm{~s}^{-1}$ and $3.3 \cdot 10^{-2} \mathrm{~s}^{-1}$, which correspond to the cross-head velocities of 5,10 and $40 \mathrm{~mm} / \mathrm{min}$, respectively. All tests are performed at room temperature on a MTS 1/ME mechanical testing machine, which is equipped with a $5 \mathrm{kN}$ load cell. A two-channel PCI-2 (Peripheral Component Interconnect), from Mistras Group, is used to record AE signals during tensile tests. All tests are performed with two Physical Acoustic resonant acoustic Pico sensors with a PAC 1220A preamplifier with $+60 \mathrm{~dB}$ gain. The peak sensitivity of the sensor is around $450 \mathrm{kHz}$ for pressure waves and $250 \mathrm{kHz}$ for the Rayleigh (surface) waves. The signals are digitized with a sampling frequency of $5 \mathrm{MHz}$. The sensors are fixed with high vacuum grease and attached to extremities of the center region of the samples with Teflon tapes. When recording AE signals, the user fixes a detection threshold ( $24 \mathrm{~dB})$, below which no signal is recorded. Time-dependent parameters, such as amplitude, energy, duration, number of counts, etc., are calculated in real-time by the acquisition system. Directly related to the shape of the waveforms, these features are widely used for AE analysis. Frequency features (e.g. average frequency) are calculated from the Fourier transform (Eq. 2).

$$
S(v)=\int s(t) e^{-2 \mathrm{i} \pi v t} d t \quad(\text { Eq. } 2)
$$

Using two sensors, our setup enables linear location of AE signals along the gauge length of the specimen: thanks to the knowledge of the velocity of the ultrasonic waves in PE samples $(V=2000 \mathrm{~m} / \mathrm{s})$ and the difference between the arrival times of a given wave at each sensor $\Delta \mathrm{t}=\left(\mathrm{t}_{1}-\mathrm{t}_{2}\right){ }^{1}$, we can get an idea of the typical distance from the sensors of the AE signal assuming it occurred on the median line joining the centers of the two sensors. Besides, only the signals

1 Comparing the measured quantity $\Delta \mathrm{t}=\left(\mathrm{t}_{1}-\mathrm{t}_{2}\right)$ to its maximum value $\Delta \mathrm{t}_{\max }=\mathrm{D} / \mathrm{V}, \mathrm{D}$ is the distance between the two sensors. Only AE signals whose $\Delta \mathrm{t}$ value is less than $\Delta \mathrm{t}_{\max }$ originates from the area between sensors where plastic deformation and/or damage of materials occur. 
whose intensity is big enough to reach both sensors can be localized and situated along the specimen. Hence, the signals are divided into two categories: the non-localized signals and the localized signals (so called "AE events"). The position $\mathrm{p}$ of those AE events is estimated according to the following relation:

$$
p=V\left(t_{1}-t_{2}\right) \quad(\mathrm{Eq} .3)
$$

\subsection{Methodology}

It seems clear from the literature that the recording of $\mathrm{AE}$ signals, from the plastic deformation of semi-crystalline polymers, is not straightforward. This could be attributed to the high attenuation of such materials. A very weak acoustic activity is generally observed. In addition, the origin of signals is not clearly identified because the localization of AE signals is not always possible. Consequently, a methodology must be conceived in order to differentiate spurious AE sources from the AE signals truly originating from plastic deformation. To do so, we chose to use two different geometries of PE samples: dumb-bell samples and "anti-samples".

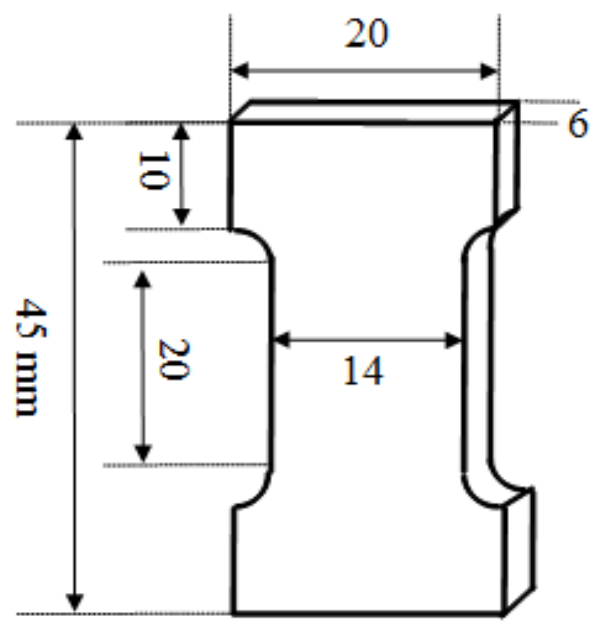

(a)

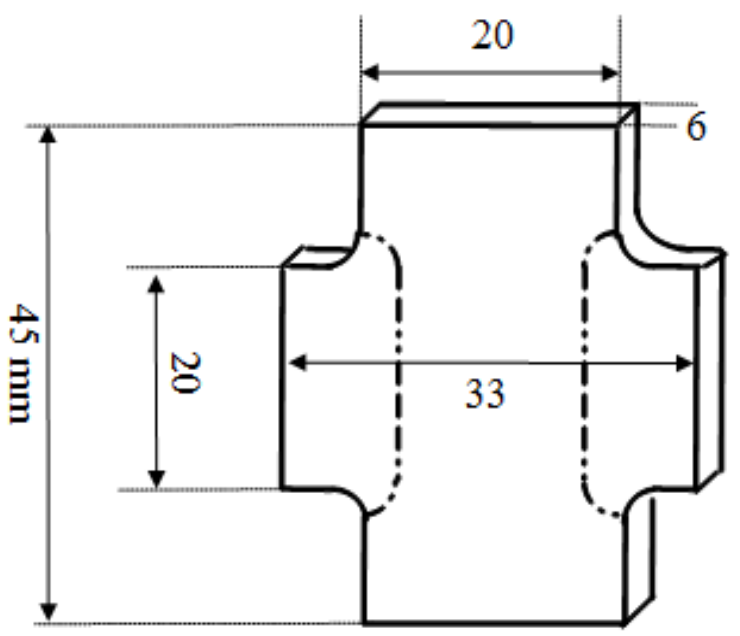

(b)

Figure. 1. Dimensions of PE (a) dumb-bell samples and (b) "anti-samples" monitored by AE technique during tensile tests. The dashed line indicates the dimensions of the dumb-bell sample on the "anti-sample".

The difference between the two samples is the width in the center region:

The dimensions of the narrow region of the dumb-bell specimens are $20 \times 14 \times 6 \mathrm{~mm}$ (see Figure. 1.a). Since the tabs are wider than the body, the stress and strain concentrate in the center region. Therefore, signals recorded during the plastic deformation and/or damage of a dumb-bell sample derive from AE sources originating from the material but also possibly from external noise, such as vibrations of the machine, or from the sliding of the tabs of samples in the grips. Hence, in order to determine the origin of the AE signals, it might be effective to use what we called "antisample" specimens.

The center region of the "anti-sample" specimens has dimensions $20 \times 33$ x $6 \mathrm{~mm}$ (see Figure. 1.b). Signals recorded cannot derive from the plastic deformation and/or damage of material. Hence, the external noise and the sliding of the tabs in the grips are the only contributions of recorded $\mathrm{AE}$ sources.

The strategy used, to partially eliminate spurious contributions (external noise and sliding) then to identify AE sources, follows two steps: 
i/ 6 pre-cycles are performed in the domain of the elastic deformation before each tensile test in order to progressively eliminate signals coming from the sliding of the tabs in the grips once the loading begins. Figure. 2.a shows an example of the apparition of spurious AE signals on the tensile curve for 6 pre-cycles performed before a tensile test at a strain rate of $4.2 \cdot 10^{-3} \mathrm{~s}^{-1}$ on a PE $\mathrm{D}_{\text {que }}$ dumb-bell sample. At each cycle, the reached load increases of $50 \mathrm{~N}$ from $1050 \mathrm{~N}$ to $1300 \mathrm{~N}$ and decreases to $200 \mathrm{~N}$ where the grips are re-tightened. The progressive elimination of AE signals deriving from the sliding of the tabs allows to prevent the recording of spurious AE signals once the tensile test begins, where, on Figure.2.a., the blue curve corresponds to the mechanical curve and blue triangles to AE signals.

ii/ Tensile tests are carried out on "anti-sample" specimens in order to identify AE signals originating from external noise and sliding, with regards to the load. Such specimens present a body wider than the tabs. The body should thus undergo only a slight elastic deformation. In addition, both dumb-bell sample and "anti-sample" tabs have the same $10 \times 20 \times 6 \mathrm{~mm}$ dimensions. Hence, their deformation is exactly the same during the tests. Thus, the recorded signals can be identified as either external noise or sliding of the tabs since the body should not be deformed. This methodology allows us to differentiate the non-localized AE signals originating from the material deformation from the spurious signals. It can be seen as a discrimination of the recording of signals, as a function of the load, in order to identify $\mathrm{AE}$ signals originating from the deformation of the material, as shown in Table 2.

\begin{tabular}{|c|l|l|l|}
\hline Specimens & \multicolumn{3}{|c|}{ AE signals } \\
\hline Dumb-bell sample & From the specimens & tabs sliding & External noise \\
\hline "Anti-sample" & & tabs sliding & External noise \\
\hline Result of the discrimination & From the specimens & \\
\hline
\end{tabular}

Table 2. Scheme of the methodology used in order to discriminate AE signals from spurious signals

Figure. 2.b shows the acoustic activity during a tensile test at the strain rate of $4.2 \cdot 10^{-3} \mathrm{~s}^{-1}$ on a $P E D_{\text {que }}$ "anti-sample" with 6 pre-cycles. The load reaches a maximum of $2450 \mathrm{~N}$ before decreasing. We notice an increase of the acoustic activity above $2000 \mathrm{~N}$, when a pronounced tabs sliding in the grips occurs: these AE signals do not come from the deformation of the material. We name this value of load, the "sliding force". Only few AE signals are detected below this "sliding force"..

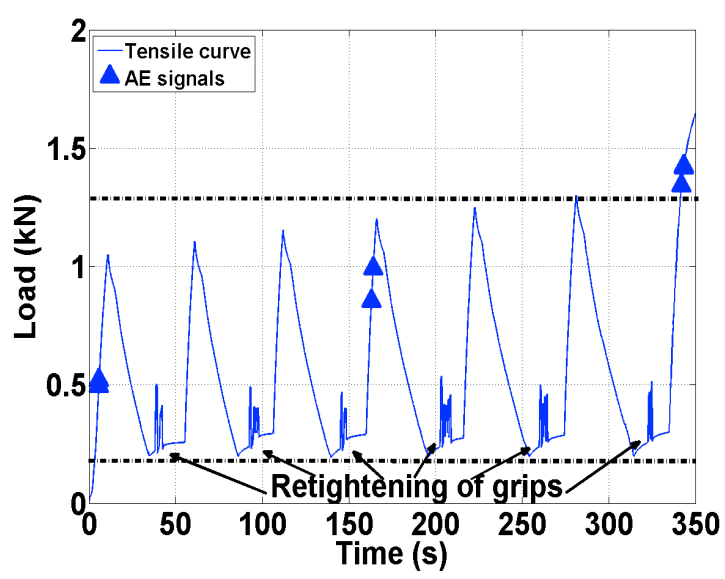

(a) 


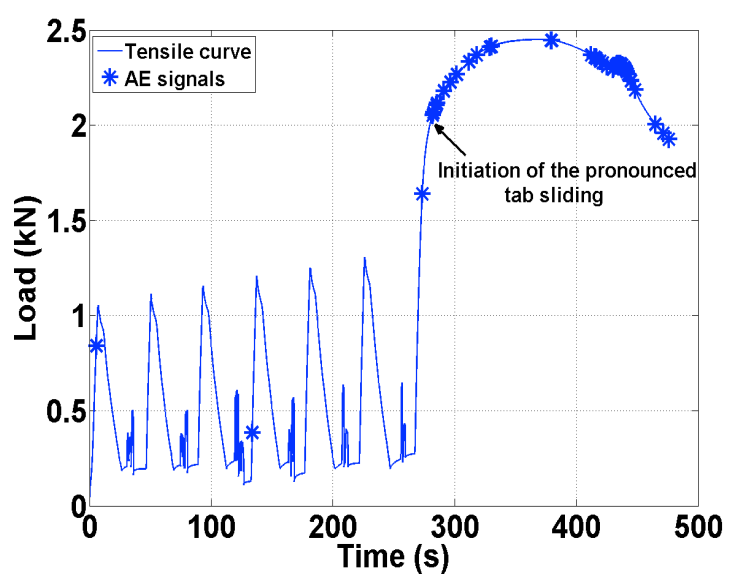

(b)

Figure. 2. (a) Acoustic activity during 6 pre-cycles before a tensile test on a PE $\mathrm{D}_{\text {que }}$ dumb-bell sample. The AE signals associated to the re-tightening of grips are not plotted. (b) Pre-cycles and tensile test at $4.2 \cdot 10^{-3} \mathrm{~s}^{-1}$ on a $P E D_{\text {que }}$ "anti-sample". The blue triangles and stars represent the $\mathrm{AE}$ signals.

\section{Ultrasonic attenuation}

Waves attenuate along their propagation throughout the medium. The dimensions of the narrow region of the dumb-bell sample minimize the attenuation of guided waves. Several contributions to the attenuation occur, such as the intrinsic attenuation of the material, the instrumentation, the beam divergence or the measurement procedure. Two types of phenomena cause intrinsic attenuation of the material: absorption and diffusion. When the acoustic wave propagates throughout the material, its absorption can be described by an exponential decay of its maximum amplitude $\mathrm{A}_{\max }$. The absorption coefficient $\alpha$ of the medium $\left(\mathrm{m}^{-1}\right)$ is generally calculated from the expression $\mathrm{A}_{\max }(\mathrm{x})=\mathrm{A}_{0} \mathrm{e}^{-\mathrm{ax}}$, where $\mathrm{x}$ is the distance between the source and the sensor $(\mathrm{m})$. Since the maximum amplitude does not consider the entire signal, it is more accurate to use the energy of the electrical signal delivered by the AE sensor, which is derived from the integral of the squared voltage divided by a reference resistance $(10 \mathrm{k} \Omega$ ) over the duration $\tau$ of the AE waveform. Hence the energy of the $\mathrm{AE}$ signal is given by the following relationship:

$$
E(x)=\frac{K}{\tau} \int_{0}^{\tau} A^{2}(x, t) d t
$$

where $K$ is a constant combining both electric and acoustic impedances. We assume that time and space variables can be separated when the propagation occurs within a non-dispersive medium. Hence, the amplitude of the wave takes the following form:

$$
A(x, t)=A_{0} e^{-\alpha x} g(t)
$$

where the function $g(t)$ represents the time dependence of the waveform. Consequently, we derive the expression of the energy $E(x)$ :

$$
E(x)=\frac{K}{\tau} \int_{0}^{\tau} A_{0}^{2} e^{-2 \alpha x} g^{2}(t) d t=\frac{K}{\tau} A_{0}^{2} e^{-2 \alpha x} \int_{0}^{\tau} g^{2}(t) d t=E_{0} e^{-2 \alpha x}
$$


with $E_{0}=\frac{K A_{0}^{2}}{\tau} \int_{0}^{\tau} g^{2}(t) d t$.

The energy $E(x)$ thus also shows an exponential decay of the form $E(x)=E_{0} e^{-\beta x}$, where the attenuation coefficient $\beta$ is:

$$
\beta=2 \alpha \quad \text { (Eq.7) }
$$

Figure. 3.a shows the exponential decay of the energy of a $500 \mathrm{kHz}$ harmonic wave as a function of the propagation distance on the surface of the $\mathrm{PE}_{\mathrm{que}}$ dumb-bell sample. Furthermore, the ultrasonic attenuation of all PE studied decreases with higher crystallinity (see Figure. 3.b). The ultrasonic attenuation of PE examined is in the range $430-790 \mathrm{~dB} / \mathrm{m}$ and can be described as a function of the crystallinity by a linear behavior. For comparison, the ultrasonic attenuation in metals is in the order ef $5-20 \mathrm{~dB} / \mathrm{m}$. Thus, the ultrasonic attenuation in semi-crystalline polymers is high. This result implies that if the energy released by the material during the tests is too weak, the

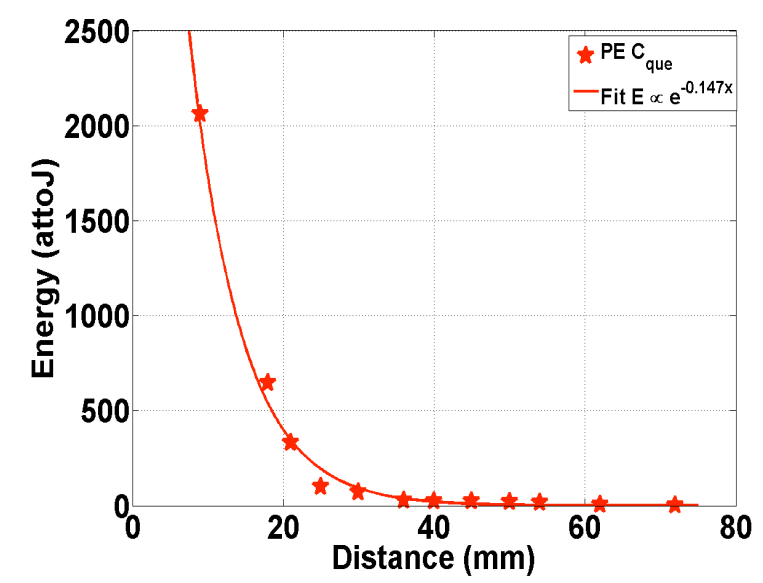

AE signal cannot be recorded on both sensors or cannot be recorded at all.

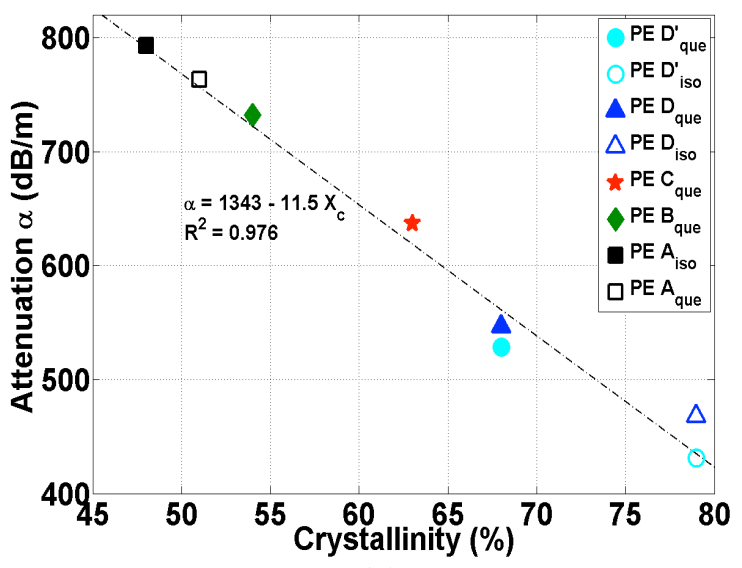

(a)

(b)

Figure. 3. (a) Energy of a $500 \mathrm{kHz}$ signal as a function of distance in the narrow region on a $\mathrm{PE} \mathrm{C}_{\text {que }}$ dumb-bell sample. The solid line corresponds to the law $\mathrm{E}^{-147 \mathrm{x}}$. (b) Attenuation of a $500 \mathrm{kHz}$ ultrasonic waves in different PE dumb-bell samples as a function of the crystallinity.

\section{Results and discussion}


In this section, we will first determine the origin of $\mathrm{AE}$ signals using the methodology with the "anti-sample" and we will present the characterization of acoustic signals during tensile tests of PE $\mathrm{D}_{\text {que }}$ dumb-bell samples. Then, we will study the influence of strain rate on the acoustic activity. Finally, we will analyze the effect of the microstructure of several PE on the AE measurements.

\subsection{Characterization and origin of sources}

To characterize the acoustic activity during a tensile test and to identify the origin of AE signals, we have first studied the PE $D_{\text {que }}$ samples. Let's recall that in order to eliminate systematically some AE spurious signals due to the initial sliding of the tabs in the grips, 6 pre-cycles are performed in the domain of the elastic deformation before each test.

Figure. 4.a shows the nominal strain-nominal stress curve with the acoustic activity for a tensile test at $4.2 \cdot 10^{-3} \mathrm{~s}^{-1}$ strain rate on a $P E \mathrm{D}_{\text {que }}$ dumb-bell sample. A particularly weak acoustic activity (around 20 signals) is collected during the tensile tests in these conditions. No AE signal is detected during the elastic deformation. Indeed, the pre-cycles eliminated the spurious signals. Some signals are recorded during the inelastic deformation, when the strain-stress dependence becomes nonlinear. Nevertheless, AE signals are observed around the yield point, which classically corresponds to the onset of plasticity of the material $[1,2]$. Hardly any AE signals are collected at the highest strain. None of those signals detected during the tests performed at a strain rate of $4.2 \cdot 10^{-3} \mathrm{~s}^{-1}$ are located on the specimen. Hence, it is difficult to conclude about the origin of these non-localized AE signals. Therefore, a comparison of the loads, at which AE signals are recorded between a dumb-bell sample and an "anti-sample", is effective to determine the origin of the acoustic signals recorded during the test. Figure. 4.b shows a comparison between the tests on a dumb-bell sample and on an "anti-sample". The load, at which the AE signals are recorded, is plotted versus the cumulative number of signals, for each sample. We notice that the sliding force which corresponds to the apparition of an intensive acoustic activity linked to the sliding of the tabs in the grips (from $2000 \mathrm{~N}$ ) is never reached in the case of a test at a $4.2 \cdot 10^{-3} \mathrm{~s}^{-1}$ strain rate on a $P E D_{\text {que }}$ dumb-bell sample. Indeed, in this last case, the maximum load reached is $1850 \mathrm{~N}$. Hence, an extensive acoustic activity, which corresponds to the strong sliding of the tabs, appears after the sliding force is reached. The recorded spurious AE signals are negligible for loads lower than the sliding force. It can be concluded that the majority of the non-localized AE signals collected derives from the material because the yield point is lower than the sliding force during tensile tests on dumb-bell samples. This result shows that the acoustic signals can be attributed to the plastic deformation and/or damage of the material with a strong certainty.

Figure. 4.c represents some characteristics of AE signals: the average frequency is plotted versus the energy. The colored crosses correspond to the maximum amplitude of each signal. The average frequency of AE signals is relatively low, in the range 160-410 kHz. The energy of the AE signals is extremely weak, in the order of a few attoJoules $\left(1\right.$ atto $\left.J=10^{-18} \mathrm{~J}\right)$, typically between 1 and 120 attoJ. The energy can reach $10^{6}$ attoJ in the case of plastic deformation and damage of steels [28]. The high attenuation of ultrasonic signals in semi-crystalline polymers could explain these weak values of recorded energy. The maximum amplitude of the recorded signals during the tests is in the range 27 to $45 \mathrm{~dB}$. The highest energy signals also present the highest amplitudes, but no correlation with the frequency content can be observed. 


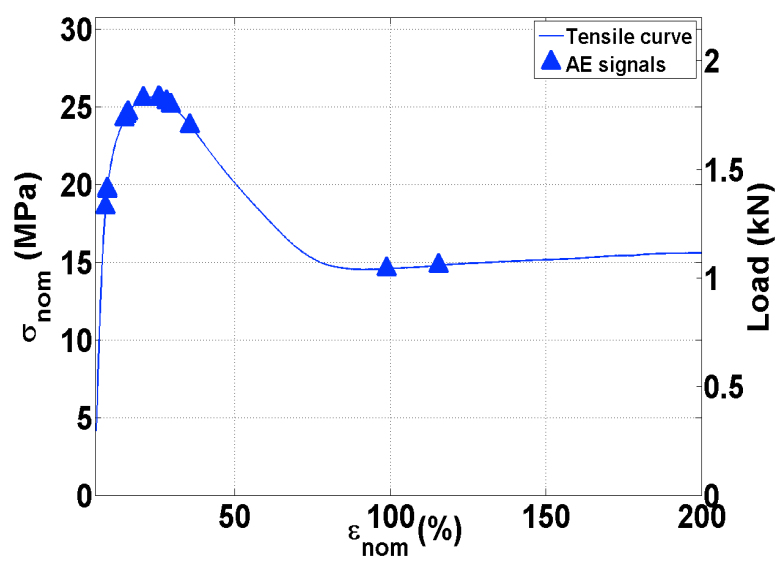

(a)

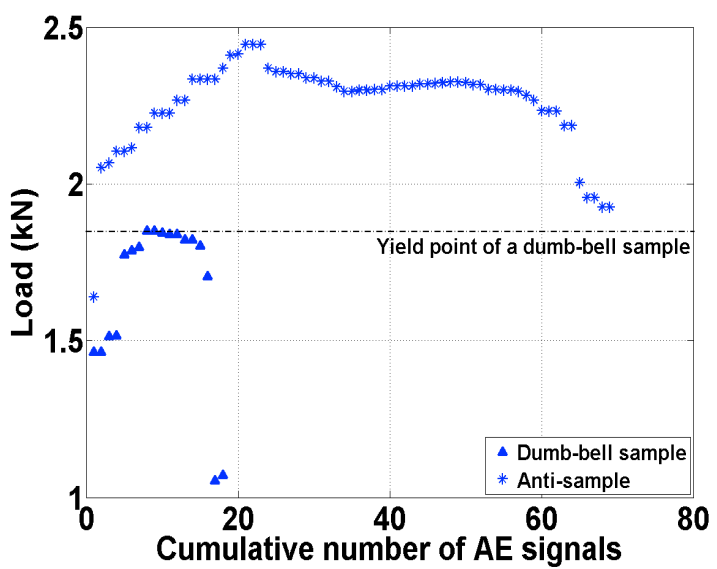

(b)

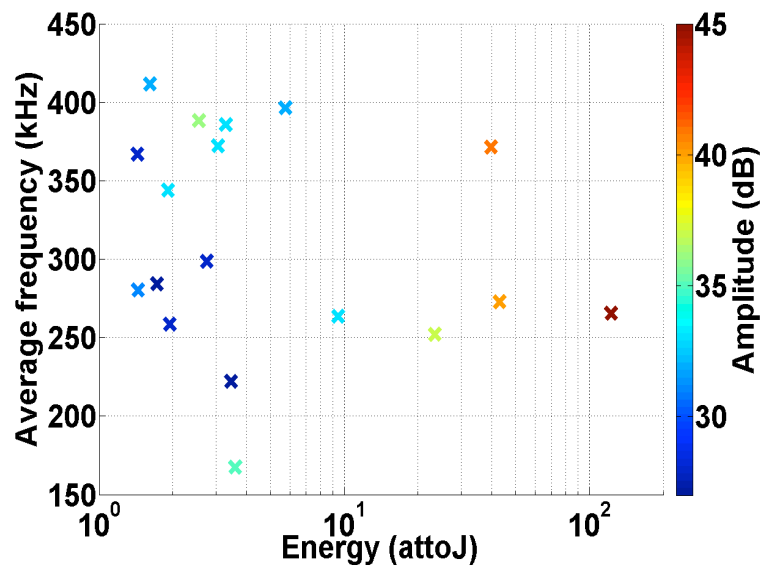

(c)

Figure. 4. (a) Acoustic activity during a tensile test on a $P E D_{\text {que }}$ dumb-bell sample. The blue curve corresponds to the tensile curve and blue triangles to the AE signals. (b) Comparison of the loads, at which the AE sources were recorded, versus the cumulative number of recorded signals for a tensile test on a dumb-bell sample (blue triangles) and an "anti-sample" (blue stars). (c) Characteristics of the recorded signals during a tensile test at a $4.2 \cdot 10^{-3} \mathrm{~s}^{-1}$ strain rate on a PE $\mathrm{D}_{\text {que }}$ dumb-bell sample. The average frequency is plotted versus the energy of the signals. The colored crosses represent the amplitude.

\subsection{Effect of strain rate}

The tests presented in the previous section were all performed at a strain rate of $4.2 \cdot 10^{-3} \mathrm{~s}^{-1}$. 
However, it is known that an increase of the strain rate has a significant effect on the recorded acoustic activity during plastic deformation and damage of metals, ceramics and composites [2931]. To evaluate the strain rate effect, we stretched the $P E D_{\text {que }}$ dumb-bell samples at three different strain rates $\left(4.2 \cdot 10^{-3} \mathrm{~s}^{-1}, 8.3 \cdot 10^{-3} \mathrm{~s}^{-1}\right.$ and $\left.3.3 \cdot 10^{-2} \mathrm{~s}^{-1}\right)$. We notice that the "sliding force" is $3000 \mathrm{~N}$ for a tensile test performed at a strain rate of $3.3 \cdot 10^{-2} \mathrm{~s}^{-1}$ on an "anti-sample".

Figure. 5.a shows the cumulative energy of AE signals and the nominal stress versus the nominal strain for three strain rates. We notice that the cumulative energy and number of AE signals increases with the strain rate. At a nominal strain of $200 \%$, the cumulative energy reaches 250,750 and 1600 attoJ for strain rates of $4.2 \cdot 10^{-3} \mathrm{~s}^{-1}, 8.3 \cdot 10^{-3} \mathrm{~s}^{-1}$ and $3.3 \cdot 10^{-2} \mathrm{~s}^{-1}$, respectively. This result could be due to the fact that more microstructural events corresponds higher energy signals. Hence, an increase of the strain rate promotes the recording of a higher number of AE signals and higher energy signals. In addition, a significant part of the energy increases around the yield point for each test. Classically, it is considered that the initiation of plastic deformation appears at the yield point. These signals must probably derive from the plastic deformation of the crystal. It is also interesting to note that some AE signals are recorded at highest strains for strain rates $8.3 \cdot 10^{-3} \mathrm{~s}^{-1}$ and $3.3 \cdot 10^{-}$ ${ }^{2} \mathrm{~s}^{-1}$ and their energy is relatively high.

We notice that some AE events are recorded during the tests at the highest strain rate $\left(3.3 \cdot 10^{-2} \mathrm{~s}^{-1}\right)$, as shown in Figure. 5.b. These AE events appear just before the yield point and during the neckpropagation along the narrow region. In these conditions, a lot of micro-mechanisms are associated to AE signals and their localization become possible. These events can be attributed with certainty at the initiation of the plasticity and/or damage of the material and bring out that the plastic deformation begins before the yield point.

The acoustic activity presents three different behaviors during a tensile test:

- At small strains (less than $70 \%$ ), more than half of the total number of AE signals (whether they are localized or not) is recorded. Although, the sensors are distant, the energy released by the plastic events is initially strongly concentrated in the samples and corresponds to the sum of a lot of AE sources. Hence, the detection of AE signals is easier (see Figure. 6.a). In addition, the majority of the acoustic activity is recorded around the yield point.

- At nominal strain in the range 70-150\%, the acoustic activity is very weak for each strain rate. Furthermore, no AE event is recorded after a nominal strain of $70 \%$. The formation of a necking region appears during this phase thus, the released energy becomes strongly diffuse in the sample (see Figure.6.b). Furthermore, the sensors are still far from the AE sources, thus the detection of AE signals is unlikely.

- At high strain, the acoustic activity increases again. The signals emitted by the plastic events are again recorded once again since the necking regions approach the sensors (see Figure. 6.c). However, it is difficult to know if these signals, whose energy is relatively high compared to others, come from high-energy damage mechanisms such as fibrillar or fracture of fibrils taking place far from the sensors, or from mechanisms close to sensors and whose energy is weakly attenuated by the short propagation in the material. 


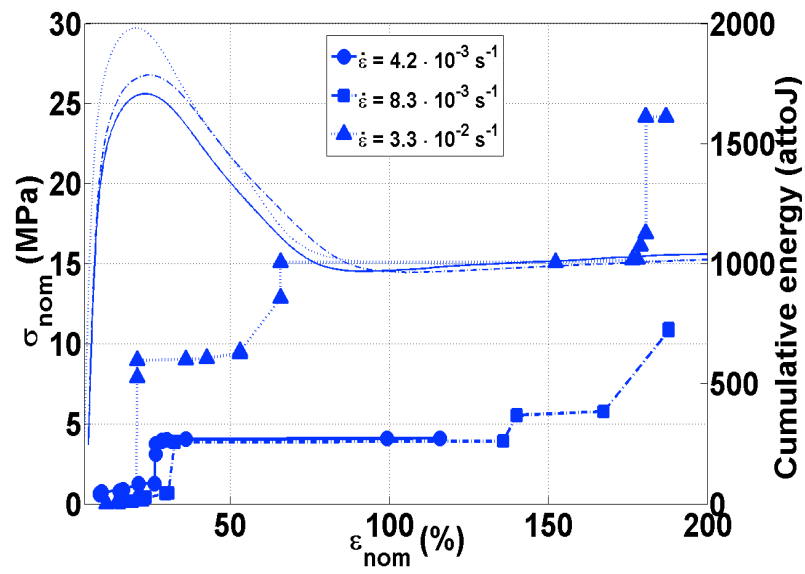

(a)

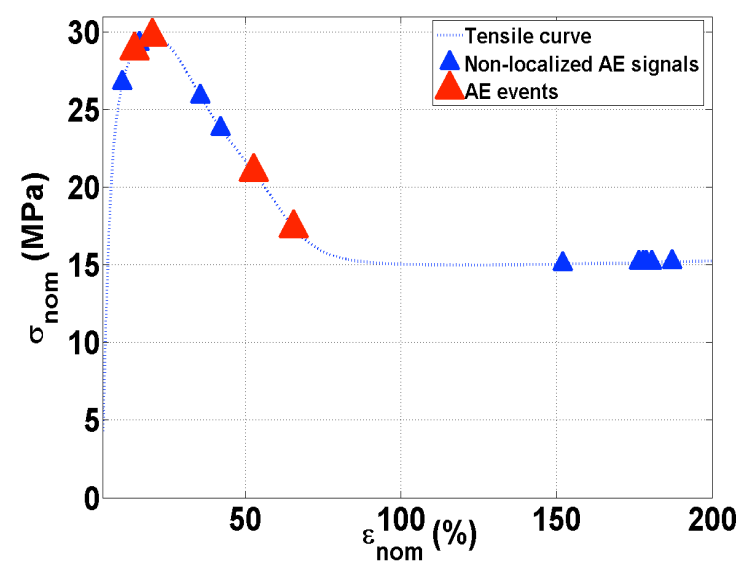

(b)

Figure. 5. (a) Cumulative energy of all AE signals for three strain rates $4.2 \cdot 10^{-3} \mathrm{~s}^{-1}$ (dots), $8.3 \cdot 10^{-}$ ${ }^{3} \mathrm{~s}^{-1}$ (squares) and $3.3 \cdot 10^{-2} \mathrm{~s}^{-1}$ (triangles). (b) Acoustic activity during a tensile test on a PE $\mathrm{D}_{\text {que }}$ dumb-bell sample at a strain rate of $3.3 \cdot 10^{-2} \mathrm{~s}^{-1}$, the blue dotted curve corresponds to the tensile curve, blue triangles to the non-localized AE signals and big red triangles to the AE events.

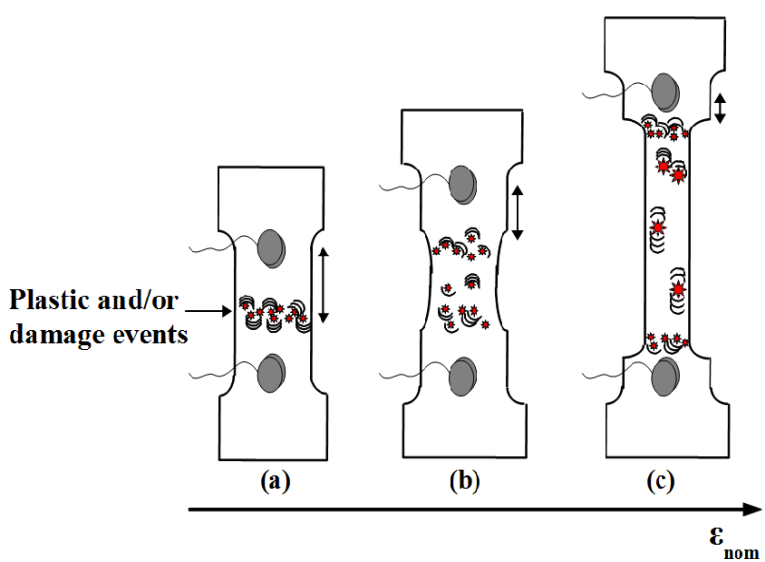

Figure. 6. Scheme of the distribution of AE signals on a dumb-bell sample during a tensile test.

\subsection{Effect of the microstructure and micro-mechanisms associated to AE signals}

We noticed, from the tests presented previously, that the acoustic activity was more substantial for higher strain rates. Therefore, it would be more interesting to characterize the plastic deformation and damage of PE samples at a strain rate of $3.3 \cdot 10^{-2} \mathrm{~s}^{-1}$. However, the number of recorded AE 
signals is still relatively weak during a test thus we chose to analyze the acoustic activity coming from 3 tensile tests performed in the same conditions. In this section, we will study the influence of the microstructure for different $\mathrm{PE}$, in particular the effect of the degree of crystallinity and the mechanism of formation of micro-cavities, on the acoustic activity. Let's recall that the mechanism of formation of cavitation is not the same for all the samples. The PE $\mathrm{A}_{\text {que }}$ and PE $\mathrm{B}_{\text {que }}$ samples do not exhibit any formation of micro-cavities. The $\mathrm{PE} \mathrm{A}_{\text {iso }}, \mathrm{PE}_{\text {que }}, \mathrm{PE}_{\text {que }}$ and $\mathrm{PE} \mathrm{D}_{\text {que }}^{\prime}$ samples exhibit a formation of micro-cavities after the yield point. The $\mathrm{PE} \mathrm{D}_{\text {iso }}$ and $\mathrm{PE} \mathrm{D}_{\text {iso }}^{\prime}$ samples exhibit a formation of cavitation before the yielding.

Figure. 7.a shows the cumulative number of AE signals (from 3 tensile tests) and the nominal stress as a function of the nominal strain for the eight examined PE samples. The mechanical curves show that the yield stress increases strongly with the crystallinity. The cumulative number of recorded AE signals (and events) increases significantly with the crystallinity. This result shows that the average number of recorded AE signals per test is 12 for PE $\mathrm{A}_{\text {que }}$, the lowest crystallinity PE, and it is 24 and 29 for PE $D_{\text {iso }}$ and PE $D_{\text {iso }}^{\prime}$, the highest crystallinity PE (see Figure. 8). We notice, in Figure. 7.a, that the behavior of the acoustic activity is the same for all PE. The recording of AE signals is initiated during the inelastic deformation, before the yield point. The significant part of the acoustic activity is collected at small strains, before $50 \%$. Then, hardly any AE signals are observed before a new increase of the acoustic activity at high strains for each studied PE. Figure. 7.b shows the cumulative number of $\mathrm{AE}$ signals at small strain. We notice that the detection of $\mathrm{AE}$ signals increases with the crystallinity, in particular for the highest crystallinities PE. Furthermore, the preyield acoustic activity is also correlated to the crystallinity.

This could be partly correlated to the cavitation, which appears before the yield point for the PE $D_{\text {iso }}$ and PE $D_{\text {iso }}^{\prime}$ samples. Similarly, the relatively high acoustic activity after yielding for PE $A_{\text {iso }}$, $\mathrm{PE} \mathrm{C}_{\text {que }}, \mathrm{PE} \mathrm{D}_{\text {que }}^{\prime}$ and $\mathrm{PE}_{\text {que }}$ samples can be partially attributed to the cavitation. The detection of $\mathrm{AE}$ signals before yielding shows that at least another micro-mechanism of deformation than the cavitation releases acoustic waves. This is brought out by $\mathrm{AE}$ measurements on $\mathrm{PE} \mathrm{A}_{\text {que }}$ and $\mathrm{PE} \mathrm{B}_{\text {que }}$ samples, which do not cavitate, where only a few AE signals are recorded (no event is recorded) before the yielding. Hence, it is clear that a second micro-mechanism of deformation releases acoustic waves. In these previous cases, the AE signals appear near the yield point, which is generally considered as the beginning of the plastic deformation of crystal $[1,2]$. Hence, these signals could be associated to the initiation of shearing of crystalline lamellae or to the fragmentation of the crystalline network.

The average energy of AE signals is also correlated with the crystallinity, as shown in Figure 9. The average energy increases strongly with the crystallinity and is in the range 10-390 attoJ. It can be suggested that the formation of cavitation comes along with a release of relatively high energy. Hence, it seems clear that the cavitation has a strong effect on the acoustic activity and the energy of AE signals.

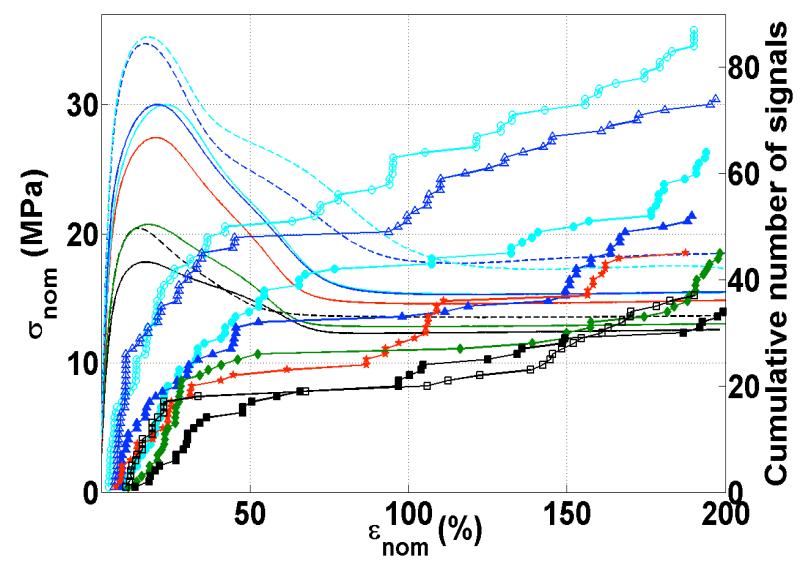


(a)

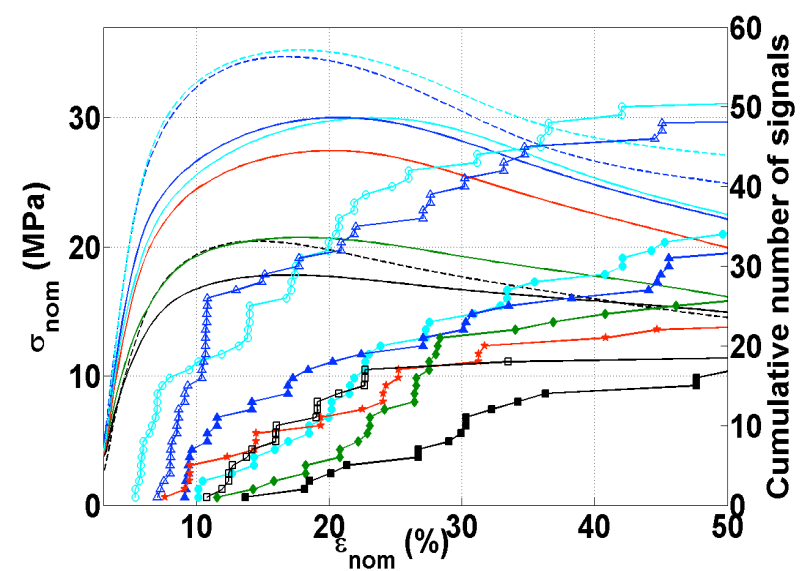

(b)

Figure. 7. (a) Comparison of the cumulative number of AE signals of studied PE at a $3.3 \cdot 10^{-2} \mathrm{~s}^{-1}$ strain rate in the range $0-200 \%$. Solids and dash-dots curves correspond to the tensile curves and symbols to the AE signals. The colors and symbols are the same as in Table 1. (b) Comparison of the cumulative number of AE signals of studied PE in the range $0-50 \%$.

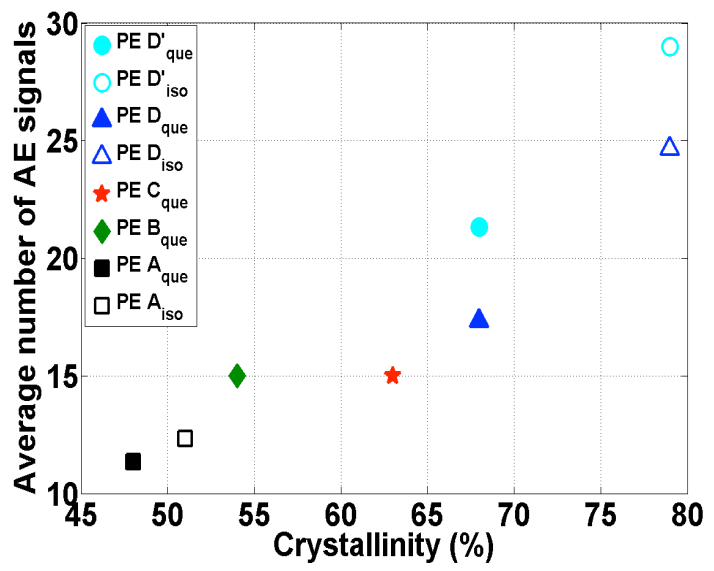

Figure. 8. Average number of AE signals per test of studied PE.

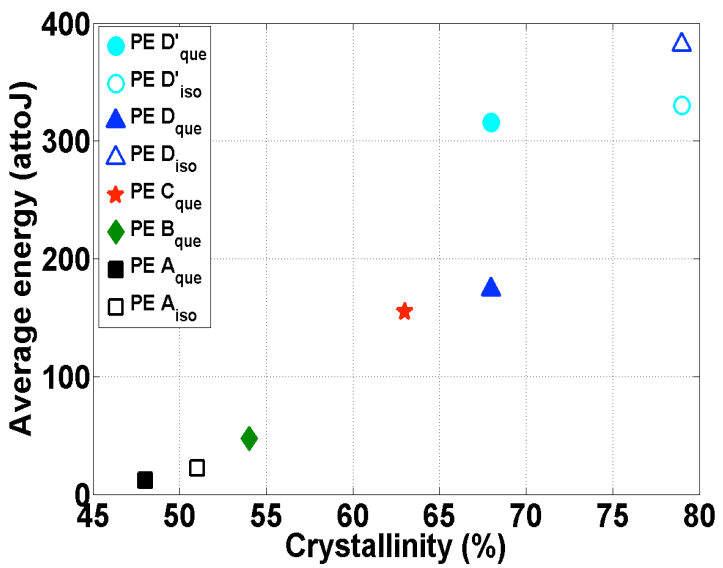

Figure. 9. Average energy of recorded AE signals of studied PE.

\section{Conclusion}

In this work, we propose to use the $\mathrm{AE}$ technique for the characterization of the initiation of 
plasticity and/or damage of several PE samples during tensile tests at room temperature. It has been shown that PE samples highly attenuate ultrasonic waves. This could explain the modest number of papers in the literature dealing with $\mathrm{AE}$ in such materials. Indeed, a weak acoustic activity is generally observed and the localization of AE signals is not always possible. Hence, it is difficult to conclude about the origin of recorded AE signals for such signals.

Thus, we have used two different methodologies in order to identify the origin of AE sources. A first source of spurious signals has been identified as the initial sliding of the tabs of the samples in the grips once the loading begins. The first methodology aimed to eliminate these signals by performing 6 pre-cycles in the elastic domain before each tensile test. The second methodology allowed us to evaluate the amount of spurious signals generated by the sliding of the tabs in the grips and the vibration of machine during tensile tests by the use of an "anti-sample", which exhibits only a little elastic deformation. In the case of tests on the "anti-sample", hardly any spurious signals are recorded below the maximal load reached during tests on dumb-bell samples. Hence, in the case of tensile tests on dumb-bell samples, most of non-localized AE signals have a strong probability to originate from the plastic deformation and/or damage of materials. However, a weak acoustic activity is collected during the tests and the localization of an amount of AE signals is only possible at high strain rates. Furthermore, the number and the energy of AE signals increase with strain rate.

We have shown that the acoustic activity could be described in 3 parts during the tests. The majority of AE signals is recorded, at small strains. In particular, the detection of signals before yielding shows that the initiation of plasticity and/or damage is initiated in the earlier stage of the deformation. A very weak acoustic activity is collected at medium strains, where the formation of a necking appears and the release of the acoustic energy becomes very diffuse in the samples. Hence, the detection of AE signals is unlikely. The number of recorded AE signals increases again at high strains, when the necking approaches the sensors.

The influence of the degree of crystallinity and the mechanisms of formation of micro-cavities has also been studied. We observed that the cumulative number of AE signals strongly increase with the crystallinity. Furthermore, the detection of AE signals before yielding is also correlated to the crystallinity. Indeed, a significant acoustic activity is collected before the yield point for the highest crystallinities PE samples, in particular for PE samples exhibiting the formation of cavitation before yielding. This result shows that micro-mechanisms of plasticity and/or damage appear before yielding and the formation of cavitation can be strongly correlated with the recording of AE signals. PE samples that do not cavitate exhibit a weak acoustic activity. In these cases, the recording of $\mathrm{AE}$ signals around the yield point suggests that the shearing of crystallites and/or the fragmentation of crystalline lamellae and/or the martensitic transformation can also be related to the detection of AE signals. In addition, the average energy of AE signals increases significantly with the crystallinity, in particular for PE samples exhibiting a formation of micro-cavities. It can be suggested that the formation of cavitation is associated with the release of a relative high energy.

\section{References}

[1] Pawlak, A. Polymer, 2007, 48, 1397-1409.

[2] Galeski, A. Prog. Polym. Sci. 2003, 28, 1643-1699.

[3] G'sell, C.; Dahoun, A. Mat. Sci. Eng. A-Struct., 1994, 175, 183-199.

[4] Bartczak, Z.; Galeski, A. Macromol. Symp. 2010, 294-I, 67-90.

[5] Lin, L.; Argon, A.S. J. Mater . Sci., 1994, 29, 294-323.

[6] G'sell, C.; Jonas, J.J. J. Mater. Sci., 1981, 16, 1956-1974.

[7] Schultz, J.M. Polym. Eng. Sci., 1984, 24, 770-785. 
[8] Seguela, R.; Staniek, E.; Escaig, B.; Fillon, B. J. Appl. Polym. Sci. 1999, 71, 1873-1885.

[9] Humbert, S.; Lame, O.; Chenal, J.M.; Rochas, C.; Vigier, G. J. Polym. Sci. Pol. Phys., 2010, 48, $1535-1542$.

[10] Humbert, S.; Lame, O.; Seguela, R.; Vigier, G. Polymer, 2011, 52, 4899-4909.

[11] Humbert, S.; Lame, O.; Chenal, J.M.; Rochas, C.; Vigier, G. Macromolecules, 2010, 43, 72127221.

[12] Xiong, B.; Lame, O.; Chenal, J.M.; Rochas, C.; Seguela, R.; Vigier, G. Polymer, 2013, 54, 5408-5418.

[13] Pawlak, A.; Galeski, A. Rozanski, A. Prog. Polym. Sci., 2014, 39, 921-958.

[14] Andre, S.; Baravian, C.; Renault, N.; Cunat, C. Appl. Phys. Lett, 2007, 91 (7).

[15] Blaise, A.; Baravian, C.; André, S.; Dillet, J.; Michot, L.; Mokso, R. Macromolecules, 2010, 43 (19), 8143-8152.

[16] Wadley, H. N. G.; Scruby, C. B.; Speake, J. H. Int. Mater. Rev., 1980, 25, 41-64.

[17] Haidar, K.; Pijaudier-Cabot, G.; Dubé, J.F.; Loukili, A. Mater. Struct., 2005, 38, 201-210.

[18] Kaita, I.; Manabu, E. Mater. T., 2007, 48, 1221-1226.

[19] Betteridge, D.; Connors, P.A.; Lilley, T.; Shoko, N.R.; Cudby, M.E.A. Polymer, 1983, 24, 1206-1212.

[20] Betteridge, D.; Connors, P.A.; Lilley, T.; Shoko, N.R.; Cudby, M.E.A. Polymer, 1982, 23, $178-184$.

[21] Grabec, I.; Peterlin, A. Polymer, 1976, 14, 651-661.

[22] Ronkay, F.; Czigány, T. Polym. Bull., 2006, 57, 989-998.

[23] Bohse, J. Compos. Sci. Technol., 2000, 60, 1213-1226.

[24] Qian, R.; Wang, T.; Shen, J. Chinese Journal of Polymer Science, 1983, 2,168-175.

[25] Galeski, A.; Koenczoel, L.; Piorkowska, E.; Baer, E. Nature, 1987, 325, 40-41.

[26] Galeski, A.; Koenczoel, L.; Piorkowska, E.; Baer, E. J. Polym. Sci. Pol. Phys., 1990, 28, 1171-1186.

[27] Wunderlich, B. Macromolecular physics: Crystal structure, morphology, defect, 1973, 1.

[28] Shaira, M.; Godin, N.; Guy, P.; Vanel, L.; Courbon, J. Mat. Sci. Eng. A, 2008, 492, 392-399.

[29] Rotem, A. Composites, 1978, 9, 33-36.

[30] Dornfeld, D.A.; Kannatey-Asibu, E. Int. I. Mech. Sci., 1980, 22, 285-296.

[31] Hamstad, M.A.; Mukherjee, A.K. Exp. Mech., 1974, 14, 33-41.

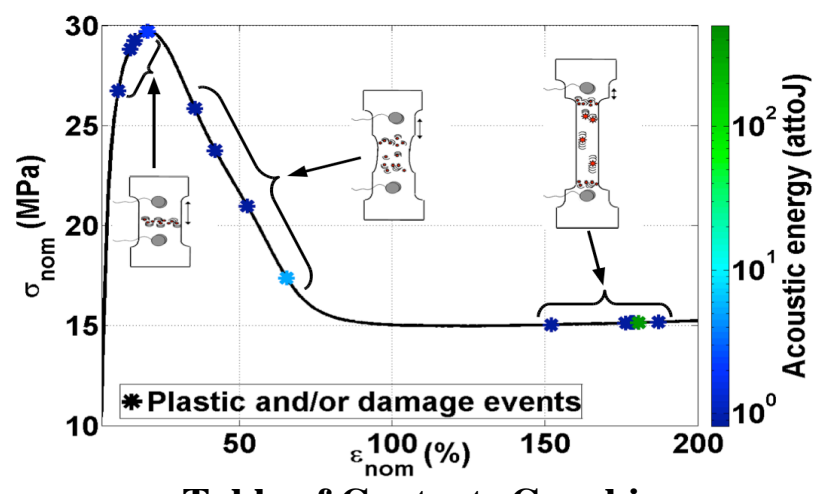

Table of Contents Graphic

Title : Acoustic emission from the initiation of the plastic deformation of Polyethylenes during tensile tests

Authors : N. Casiez, S. Deschanel, T. Monnier, and O. Lame 